\title{
Detection of Decarboxylated Amino Acids after in Vitro Protease Digestion of the Hydrophilic Fraction of Crude Drug Extracts
}

\author{
Saki Shirako, ${ }^{a}$ Kenji Sato, ${ }^{b}$ Saki Moriwaki, ${ }^{a}$ Yukinobu Ikeya, ${ }^{*, c}$ and Mikio Nishizawa ${ }^{a}$ \\ ${ }^{a}$ Department of Biomedical Sciences, College of Life Sciences, Ritsumeikan University; Kusatsu, Shiga 525-8577, \\ Japan: ${ }^{b}$ Division of Applied Biosciences, Graduate School of Agriculture, Kyoto University; Kyoto 606-8502, \\ Japan: and ${ }^{c}$ Center for Supporting Pharmaceutical Education, Daiichi University of Pharmacy; 22-1 Tamagawa-cho, \\ Minami-ku, Fukuoka 815-8511, Japan.
}

Received July 28, 2021; accepted November 29, 2021

\begin{abstract}
Many constituents of crude drugs in Japanese Kampo formulas are thought to function as pro-drugs, whose pharmacological activity is manifested after oral administration. Proteins and peptides in crude drugs may be digested and metabolized in the digestive tract and liver. However, few studies have reported the pharmacological activity of peptides in crude drugs. Here, we applied an analysis using LC-tandem mass spectrometry (LC-MS/MS) to identify the compounds derived from six crude drugs that are assumed to have anti-inflammatory effects. To simulate in vivo protease digestion, each water-soluble fraction of the crude drug extracts was treated with proteases, including endoproteinases and exopeptidases. Amines in the resultant digests were modified by 6 -aminoquinolyl- $N$-hydroxysuccinimidyl carbamate and analyzed using LC-MS/MS, which demonstrated the presence of four decarboxylated amino acids (primary amines). In the digest of the hydrophilic fraction of the fruit of Ziziphus jujuba Miller var. inermis Rehder (Taiso), isobutylamine, isoamylamine, and 2-methylbutylamine were identified, which may be derived from valinyl, leucinyl, and isoleucinyl residues, respectively. Additionally, tyramine possibly derived from tyrosyl residues was identified in the digests of all the crude drugs. In primary cultured rat hepatocytes treated with interleukin-1 $\beta$, all these decarboxylated amino acids suppressed the production of nitric oxide, a proinflammatory mediator. Our approach, i.e., in vitro protease digestion and LC-MS/MS analysis, suggests that decarboxylated amino acids may be formed in vivo from peptides and may be responsible for the anti-inflammatory effect of crude drugs included in Kampo medicine.
\end{abstract}

Key words in vitro protease digestion; peptidase; LC-tandem mass spectrometry (LC-MS/MS); branchedchain amino acid; Kampo medicine

\section{INTRODUCTION}

Traditional Japanese medicine, i.e., Kampo drugs, possesses a variety of pharmacological effects. It is intriguing to study how Kampo drugs exert their activities in the body. Crude drugs included in Kampo formulas consist of many constituents, which can be fractionated from an extract into hydrophobic, amphipathic, and hydrophilic fractions. ${ }^{1)}$ Many hydrophobic constituents in ethyl acetate-soluble fractions of extracts from crude drugs, such as limonin and obakunone in the bark of Phellodendron amurense, sakuranetin and (-)-naringenin in the bark of Prunus jamasakura, and phthalides in the rhizome of Cnidium officinale, have been investigated for their pharmacological activities. ${ }^{2-4)}$ Primary cultured rat hepatocytes have been used as an ex vivo system to mimic liver injury, ${ }^{5)}$ and nitric oxide (NO), a proinflammatory mediator, ${ }^{6}$ is produced from hepatocytes. Because the suppression of interleukin (IL) $1 \beta$-induced NO production is correlated with the anti-inflammatory activity of a constituent, the anti-inflammatory potency of a constituent can be measured by using IL-1 $\beta$-treated hepatocytes. ${ }^{7)}$

Glycosides in the $n$-butanol-soluble (amphipathic) fraction from crude drug extracts have also been studied and are considered to function as pro-drugs. ${ }^{8)}$ For example, sennosides were hydrolyzed by Bifidobacterium species to promote intestinal peristalsis in mice. ${ }^{9)}$ When a crude drug is ingested, the glycosides in the crude drug are hydrolyzed by intestinal bacteria in the digestive tract and metabolized in the liver, resulting in the manifestation of its pharmacological activities in the body. Three pairs of glucosides and their aglycones in the roots and stolons of Glycyrrhiza uralensis were compared with regard to their ability to suppress NO production in hepatocytes. ${ }^{10)}$

The water-soluble (hydrophilic) fraction from methanol extracts includes peptides, amino acids, sugars, and low-molecularweight compounds. Chlorogenic acid, which is present in the hydrophilic fraction of the extract from the flower of Lonicera japonica, suppressed NO production in IL-1 $\beta$-treated hepatocytes. ${ }^{1)}$ In contrast, sugars (i.e., inositol, fructose, glucose, and sucrose) did not significantly suppress NO production. ${ }^{1)}$ Several amino acids (i.e., lysine, tryptophan, histidine, and arginine) significantly decreased both NO production and the expression of inducible nitric oxide synthase (iNOS), which synthesizes NO in hepatocytes. ${ }^{11)}$ Generally, peptides are thought to be degraded by digestion in the intestine, liver, and blood. To the best of our knowledge, there are very few studies examining the pro-drug activity of peptides in Kampo medicine.

Proteins and peptides in foods are digested by proteases from the stomach, liver, and pancreas, metabolized in the digestive tract, and finally absorbed from the intestine through the liver into the blood. Several enzymes are involved in digestion: pepsin from the stomach, pancreatic enzymes (e.g., trypsin, chymotrypsin, and carboxypeptidase A), and leucine aminopeptidase in enterocytes, bile, and pancreatic juice. ${ }^{12,13)}$ Some oligopeptides resist endoproteinases, such as pepsin and 
trypsin, but most of them are degraded by exopeptidases, such as carboxypeptidase A and leucine aminopeptidase. However, some peptides harboring specific sequences resist digestion by both proteases. ${ }^{13)}$ These oligopeptides of plant and animal origins have biological activities. ${ }^{14,15)}$ For example, pyroglutamyl leucine (pyroGlu-Leu), which is a protease-resistant dipeptide found in fermented foods, suppressed both NO production and iNOS induction in IL-1 $\beta$-treated hepatocytes. ${ }^{16)}$ PyroGlu-Leu attenuated high-fat diet-induced disturbance of intestinal microbiota (dysbiosis) by increasing host antimicrobial peptide in the intestine. ${ }^{17)}$ To simulate protease digestion in the human body and elucidate the function of digested peptides, in vitro digestion of peptides by proteases, including endoproteinases and exopeptidases, was recently performed. ${ }^{13,18)}$

Peptides, as well as their digests and metabolites formed in the digestive tract, can be detected by using LC-tandem mass spectrometry (MS/MS). As another analysis, a subset of peptides in a sample that contain specific functional groups, e.g., primary and secondary amines, can be detected by LC-MS/MS using derivatization with 6-aminoquinolyl- $N$ hydroxysuccinimidyl carbamate (AQC) and subsequent precursor ion scanning of a targeting product ion from the AQC moiety $(m / z 171.1){ }^{18)}$ Product ion scanning of the precursor ions detected by precursor ion scanning at different collision energies allows structural information.

In the current study, we selected six crude drugs that show anti-inflammatory effects, and the hydrophilic fraction of the crude drugs was digested in vitro by protease. LC-MS/MS analysis was applied to identify compounds (peptides, amino acids, and amino acid-related compounds) derived from these crude drugs. Finally, we estimated the anti-inflammatory potencies of some primary amines in the suppression of NO production in hepatocytes.

\section{MATERIALS AND METHODS}

Plant Materials and Reagents The fruit of Ziziphus jujuba Miller var. inermis Rehder (Rhamnaceae) (Taiso; collected in Shaanxi Province, China; Lot No. 7115012), the seed of Ziziphus jujuba Miller var. spinosa Hu ex H. F. Chou (Rhamnaceae) (Sansonin; collected in Hebei Province, China; Lot No. 21118002), the rhizome of Atractylodes macrocephala Koidzumi (Asteraceae) (Byakujutsu; collected in Zhejiang Province, China; Lot No. 9317006), the rhizome of Atractylodes chinensis Koidzumi (Asteraceae) (Sojutsu; collected in Shaanxi Province, China; Lot No. 6715008), the flower of Artemisia capillaris Thunberg (Compositae) (Inchinko; collected in Nagano Prefecture, Japan; Lot No. 020820002), and the root of Angelica acutiloba Kitagawa (Umbelliferae) (Toki; collected in Nara Prefecture, Japan; Lot No. 11411041) were purchased from Tochimoto Tenkaido Co., Ltd. (Osaka, Japan) and authenticated by Dr. Yutaka Yamamoto (Tochimoto Tenkaido Co., Ltd.) according to the methods described in The Japanese Pharmacopoeia. ${ }^{19)}$ The species of the Sojutsu sample was identified as Atractylodes chinensis using its genomic DNA. ${ }^{20)}$ The voucher samples were deposited in the Ritsumeikan Herbarium of Pharmacognosy, Ritsumeikan University under the code numbers RIN-ZJI-25 (Ziziphus jujuba var. inermis), ZJS-26 (Ziziphus jujuba var. spinosa), AM-27 (Atractylodes macrocephala), AC-20 (Atractylodes chinensis), ${ }^{20)}$ ACA-28 (Artemisia capillaris), and AA-29 (Angelica acutiloba). Por- cine pepsin and pancreatin, which are mixtures of pancreatic enzymes and include trypsin, were purchased from Nacalai Tesque (Kyoto, Japan). Carboxypeptidase A (CPA) from bovine pancreas and microsomal leucine aminopeptidase (LAP) from porcine kidney were purchased from Sigma-Aldrich Corp. (St. Louis, MO, U.S.A.). AQC was purchased from Toronto Research Chemicals (Toronto, ON, Canada). Isobutylamine, isoamylamine, 2-methylbutylamine, and tyramine were obtained from Tokyo Chemical Industry Co., Ltd. (Tokyo, Japan) and used as standards for LC-MS/MS analyses. Amino acids mixture standard solution (type $\mathrm{H}$, high range), valine, isoleucine, and leucine were purchased from FUJIFILM Wako Pure Chemical Corporation (Osaka, Japan).

Extraction from Crude Drugs and Fractionation Each crude drug (approximately $80 \mathrm{~g}$ ) was pulverized, extracted with methanol under reflux, and fractionated, as previously described. ${ }^{1,3,20)}$ Methanol extraction of crude drugs is the standard method to analyze their constituents. Briefly, the resultant extract was suspended in water and successively extracted with ethyl acetate and $n$-butanol (Fig. 1A). These layers were collected and concentrated by evaporation of solvents to prepare an ethyl acetate-soluble (hydrophobic) fraction, an n-butanol-soluble (amphipathic) fraction, and a water-soluble (hydrophilic) fraction. The yield of the hydrophilic fraction was $91.9 \%$ for the fruit of Ziziphus jujuba var. inermis (Taiso), $37.0 \%$ for the seed of Ziziphus jujuba var. spinosa (Sansonin), $67.7 \%$ for the rhizome of Atractylodes macrocephala (Byakujutsu), $53.3 \%$ for the rhizome of Atractylodes chinensis (Sojutsu) ${ }^{20)} 27.6 \%$ for the flower of Artemisia capillaris (Inchinko), and $82.1 \%$ for the root of Angelica acutiloba (Toki).

Protein Assay The amount of protein in the hydrophilic fraction was measured by the Bradford method using Protein Assay Coomassie Brilliant Blue Solution (Nacalai Tesque) according to the instruction manual. As a standard, bovine serum albumin was used.

In Vitro Protease Digestion The hydrophilic fraction was suspended in water and lyophilized. As control experiments, valine, isoleucine, and leucine were used instead of the hydrophilic fractions. Protease digestion was performed according to a previously published method. ${ }^{21)}$ Briefly, the resultant powder $(50 \mathrm{mg})$ was dissolved in $0.1 \mathrm{M} \mathrm{HCl}(7.5 \mathrm{~mL})$, and pepsin $(0.5 \mathrm{mg})$ was added (Fig. 1B). This reaction mixture was incubated at $37^{\circ} \mathrm{C}$ for $3 \mathrm{~h}$. Then, $1 \mathrm{M}$ Tris- $\mathrm{HCl}, \mathrm{pH} 8.0(500 \mu \mathrm{L})$, was added, and the $\mathrm{pH}$ of the mixture was adjusted by $\mathrm{NaOH}$ to 8.0. Pancreatin ( $2 \mathrm{mg}$ ) was added, and the mixture was further incubated at $37^{\circ} \mathrm{C}$ for $24 \mathrm{~h}$. After adjustment of the final volume to $10 \mathrm{~mL}$, the mixture was centrifuged at $3000 \times \boldsymbol{g}$ at $23^{\circ} \mathrm{C}$ for $10 \mathrm{~min}$. The reaction was terminated by filtration with an Amicon Ultra filter unit (molecular weight cutoff, 10000; Merck, Darmstadt, Germany) at $14000 \times \boldsymbol{g}$ at $23^{\circ} \mathrm{C}$ for $10 \mathrm{~min}$. An aliquot $(1 \mathrm{~mL})$ of this endoproteinase digest was subjected to digestion by CPA ( 6 units) and LAP (5 units) at $37^{\circ} \mathrm{C}$ for $24 \mathrm{~h}$. The reaction was stopped by ultrafiltration, and the filtrate was collected as the exopeptidase digest.

Derivatization of Amines by AQC and Precursor Ion Scanning Analysis The final concentrations of all the samples were adjusted to $5.0 \mathrm{mg}$ of hydrophilic fraction per $\mathrm{mL}$. A $150-\mu \mathrm{L}$ aliquot of each sample was dried and dissolved in $20 \mu \mathrm{L}$ of $20 \mathrm{mM} \mathrm{HCl}$. Amino acids mixture standard solution was used as the standards for the subsequent analyses. Then, primary and secondary amines were derivatized by AQC by 
A

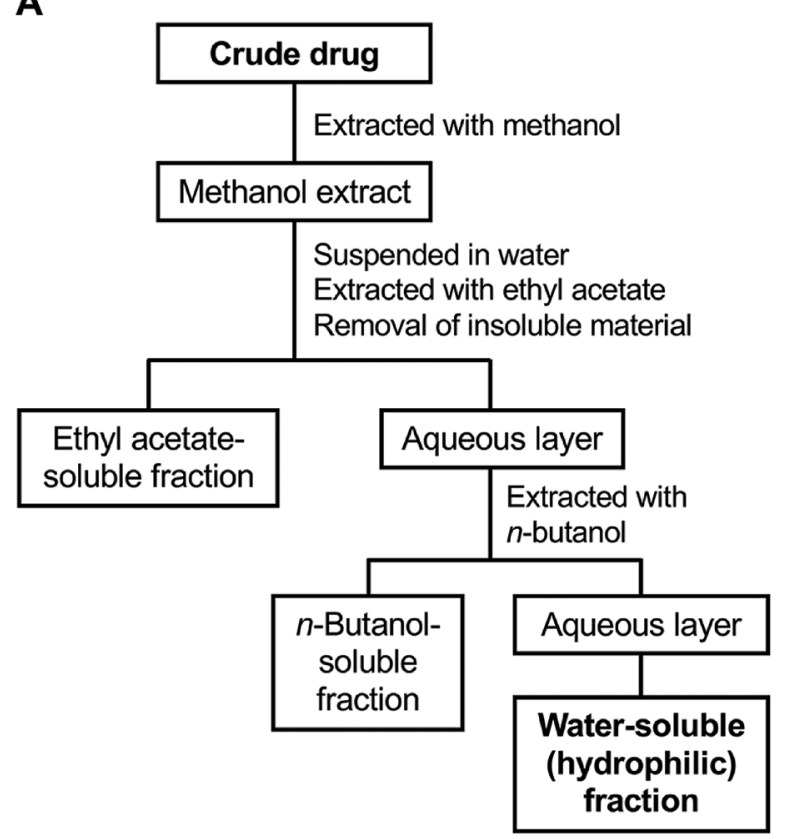

B

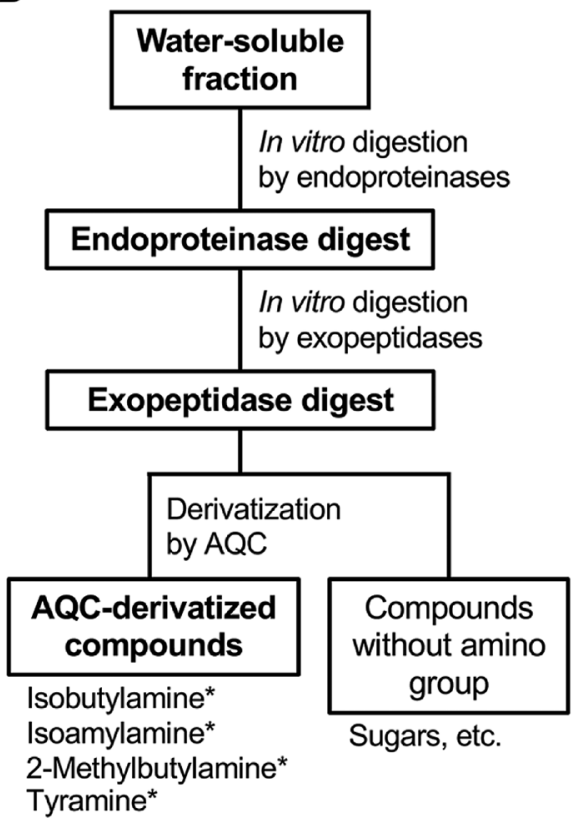

Fig. 1. Fractionation of Constituents from Crude Drugs and LC-MS/MS Analysis

(A) A flowchart of the procedures used to fractionate constituents from crude drugs. The plant material was extracted with methanol. The dried extract was suspended in water and sequentially fractionated by hydrophobicity with ethyl acetate, $n$-butanol, and water to obtain three fractions. (B) A flowchart of the procedures to identify amines in hydrophilic (water-soluble) fractions. The hydrophilic fraction was digested in vitro by proteases, including endoproteinases and exopeptidases, and the resultant digest was derivatized by 6 -aminoquinolyl- $N$-hydroxysuccinimidyl carbamate (AQC) and analyzed using LC-MS/MS. * Compounds identified in this study.

the method previously reported. ${ }^{18)}$ Briefly, after the addition of $0.3 \%$ AQC-acetonitrile solution $(20 \mu \mathrm{L})$ and $50 \mathrm{mM}$ sodium borate buffer, $\mathrm{pH} 8.8(60 \mu \mathrm{L})$, to each sample solution, the mixture was kept at $50^{\circ} \mathrm{C}$ for $10 \mathrm{~min}$. After cooling, the reactant was mixed with $100 \mu \mathrm{L}$ of $5 \mathrm{mM}$ sodium phosphate buffer, $\mathrm{pH} 7.4$, containing $5 \%$ acetonitrile and clarified by passing through a $0.45-\mu \mathrm{m}$ Cosmonice ${ }^{\circledR}$ filter (Nacalai Tesque). The filtrate $(10 \mu \mathrm{L})$ was injected into an LC-MS/MS model LCMS8040 (Shimadzu Corporation, Kyoto, Japan) equipped with an Inertsil ODS-3 column (particle size, $5 \mu \mathrm{m} ; 2.1 \mathrm{~mm}$ internal diameter $\times 250 \mathrm{~mm}$; GL Science, Tokyo, Japan).

A binary linear gradient was used with $0.1 \%$ formic acid in water (solvent $\mathrm{A}$ ) and $0.1 \%$ formic acid in $80 \%$ acetonitrile (solvent B) at a flow rate of $0.2 \mathrm{~mL} / \mathrm{min}$. The gradient program was as follows: $0-30 \mathrm{~min}, 0-30 \% \mathrm{~B} ; 30-40 \mathrm{~min}, 30-100 \% \mathrm{~B}$; 40-50 min, $100 \% \mathrm{~B} ; 50.0-50.1 \mathrm{~min}, 100-0 \% \mathrm{~B} ; 50.1-60 \mathrm{~min}$, $0 \% \mathrm{~B}$. The column was maintained at $40^{\circ} \mathrm{C}$. AQC derivatives were specifically detected by selecting precursor ions, which generated ions of the AQC-derived product (b1 ion, $\mathrm{m} / \mathrm{z}$ 171.1) in positive mode (collision energy, $-35 \mathrm{~V}$ ) in the range of $\mathrm{m} / \mathrm{z} 240-275,275-300,300-325,300-325,325-350,350-375$, 375-400, 400-450, 450-500, and 500-1000 (precursor ion scan). The $\mathrm{m} / \mathrm{z}$ of AQC-peptides was recorded and entered into the LabSolutions Ver. 5.80 software (Shimadzu Corporation). To estimate the structure of compounds in peaks, a product ion scan targeting the detected precursor ions was performed at collision energies of $-15,-25$, and $-35 \mathrm{~V}$. AQC-derivatives of standard amino acids were also prepared and analyzed by LC-MS/MS, as described above: glycine $(\mathrm{m} / \mathrm{z} 246.2$ in precursor ion scan), alanine ( $m / z 260.3)$, serine $(m / z 276.3)$, proline $(\mathrm{m} / \mathrm{z} 286.2)$, valine $(\mathrm{m} / \mathrm{z} 288.2)$, threonine $(\mathrm{m} / \mathrm{z} 290.2)$, aspartic acid $(\mathrm{m} / \mathrm{z}$ 304.2), glutamic acid $(\mathrm{m} / \mathrm{z} 318.2)$, methionine $(\mathrm{m} / \mathrm{z}$ $320.2)$, isoleucine $(\mathrm{m} / \mathrm{z} 302.3)$, leucine $(\mathrm{m} / \mathrm{z} 302.3)$, phenylalanine $(\mathrm{m} / \mathrm{z} 336.2)$, tyrosine $(\mathrm{m} / \mathrm{z} 352.3)$, and lysine (lysine de- rivatized by two AQC molecules; $m / z$ 487.4).

Quantification of Decarboxylated Amino Acids by Multiple Reaction Monitoring Aliquots $(100 \mu \mathrm{L}$ each) of the undigested hydrophilic fraction and the digests were dried and derivatized with $\mathrm{AQC}$, as described above. After the reaction, $5 \mathrm{mM}$ sodium phosphate buffer, $\mathrm{pH} 7.4$, containing 5\% acetonitrile $(50 \mu \mathrm{L})$ was added to the reactant and clarified by passing through a $0.45-\mu \mathrm{m}$ filter. The filtrate $(10 \mu \mathrm{L})$ was injected into an LCMS8040 equipped with an Inertsil ODS-3 column. The contents of the AQC-decarboxylated amino acids in the reaction mixture were quantified via LC-MS/MS in multiple reaction monitoring (MRM) mode using LabSolutions software. The elution conditions were the same as those of precursor and product ion scanning. Isobutylamine, isoamylamine, 2-methylbutylamine, and tyramine were dissolved in methanol and used as standards for the optimization of MRM conditions. The calibration equations and correlation coefficients of three standard compounds were as follows: isobutylamine, $y=9000000 x+116323\left(R^{2}=0.9999\right.$; the fruit of Ziziphus jujuba var. inermis) and $y=9000000 x+25571$ ( $R^{2}=0.9999$; the rhizome of Atractylodes macrocephala); 2-methylbutylamine, $y=10000000 x+53398 \quad\left(R^{2}=0.9999\right)$; isoamylamine, $y=10000000 x+113517 \quad\left(R^{2}=1.0000\right)$. The peak areas of the base peak ions of isobutylamine, 2-methylbutylamine, isoamylamine, and tyramine in the sample solution were fit to the calibration curves. The content was calculated as each decarboxylated amino acid (pmol) divided by the dried hydrophilic fraction (mg).

Animal Experiments and Primary Cultured Rat Hepatocytes All animal care and experimental procedures were performed in accordance with the laws and guidelines of the Japanese government and were approved by the Animal Care Committee of Ritsumeikan University, Biwako-Kusatsu Campus. Male Wistar rats (Charles River Laboratories Japan, 
Inc., Yokohama, Japan) were housed at $21-23^{\circ} \mathrm{C}$ under a $12-\mathrm{h}$ light-dark cycle and fed a CRF-1 diet (Charles River Laboratories Japan) with water available ad libitum. The animals were acclimated to their housing for at least one week. Hepatocytes were isolated from the livers of Wistar rats, as previously described. ${ }^{22)}$ Briefly, the liver was perfused with collagenase, and the dispersed cells were centrifuged, resuspended, and seeded at $1.2 \times 10^{6}$ cells per $35-\mathrm{mm}$ diameter dish. The cells were incubated at $37^{\circ} \mathrm{C}$ for $2 \mathrm{~h}$. After the medium was changed, the hepatocytes were further incubated at $37^{\circ} \mathrm{C}$ overnight.

Estimation of NO Production and Lactate Dehydrogenase (LDH) Activity Each fraction or compound was added to the medium on day 1 , and the hepatocytes were incubated for $8 \mathrm{~h}$. Nitrite (a stable metabolite of NO) in the medium was measured using the Griess method to measure NO concentrations. ${ }^{23,24)}$ The NO levels in the presence and absence of IL- $1 \beta$ in the medium were set at 100 and $0 \%$, respectively. The $\mathrm{IC}_{50}$ against nitrite was determined in triplicate for at least three different concentrations of an extract, a fraction, or a compound. ${ }^{24)}$ LDH activity in the medium was measured using an LDH Cytotoxicity Detection Kit (TaKaRa Bio Inc., Kusatsu, Japan) to estimate cytotoxicity. The $\mathrm{IC}_{50}$ value of a compound was calculated to determine its ability to inhibit NO production when the compound did not show cytotoxicity.

Direct NO Quenching Activity Each compound was added to a medium containing $25 \mu \mathrm{M} \mathrm{NaNO}$ and incubated at $37^{\circ} \mathrm{C}$ for $1.5 \mathrm{~h}$, according to a previously published method. ${ }^{10)}$ The final concentration of each compound was $4.0 \mathrm{mM}$ (isobutylamine), $3.0 \mathrm{mM}$ (2-methylbutylamine), $2.5 \mathrm{mM}$ (isoamylamine), and $0.80 \mathrm{mM}$ (tyramine). This medium was then mixed with Griess reagent ${ }^{23,24)}$ and incubated at $23^{\circ} \mathrm{C}$ for $5 \mathrm{~min}$. The absorbance at $540 \mathrm{~nm}$ was measured to determine the reduction of nitrite by the compound.

Statistical Analysis The results are representative of at least three independent experiments that gave similar findings. The values are presented as the mean \pm standard deviation
(S.D.). The differences were analyzed using Student's $t$-test followed by Bonferroni correction. The statistical significance was set at $p<0.05$ and $p<0.01$.

\section{RESULTS}

In Vitro Protease Digestion of the Water-Soluble Fraction of Crude Drug Extracts Crude drugs that are considered to possess anti-inflammatory effects were used for methanol extraction. The six crude drugs used in this study were the fruit of Ziziphus jujuba var. inermis (Taiso), the seed of Ziziphus jujuba var. spinosa (Sansonin), the rhizome of Atractylodes macrocephala (Byakujutsu), the rhizome of Atractylodes chinensis (Sojutsu), the flower of Artemisia capillaris (Inchinko), and the root of Angelica acutiloba (Toki). NO production was measured in IL- $1 \beta$-treated hepatocytes when an extract from each crude drug was added to the medium. As expected, the extracts from five crude drugs suppressed NO production, whereas the effect of the extract from the seed of Ziziphus jujuba var. spinosa on NO production could not be evaluated due to its cytotoxicity (data not shown).

Then, the methanol extracts were successively partitioned into three fractions based on hydrophobicity using ethyl acetate, $n$-butanol, and water (Fig. 1). To simulate the in vivo digestion of crude drugs, the water-soluble (hydrophilic) fractions of the crude drug extracts were subjected to protease digestion. The protein content in the dried hydrophilic fractions ranged from $15.9 \mu \mathrm{g}$ per mg (the fruit of Ziziphus jujuba var. inermis and the rhizome of Atractylodes chinensis) to $37.2 \mu \mathrm{g}$ per mg (the seed of Ziziphus jujuba var. spinosa).

Detection of Amines after In Vitro Protease Digestion of Crude Drug Extracts Total ion chromatograms of the protease digests showed many peaks at the scan range of $\mathrm{m} / \mathrm{z}$ from 240 to 375 (data not shown). Because this range corresponds to the $m / z$ of AQC-derivatized amino acids, protein in the hydrophilic fraction of the crude drugs may be completely

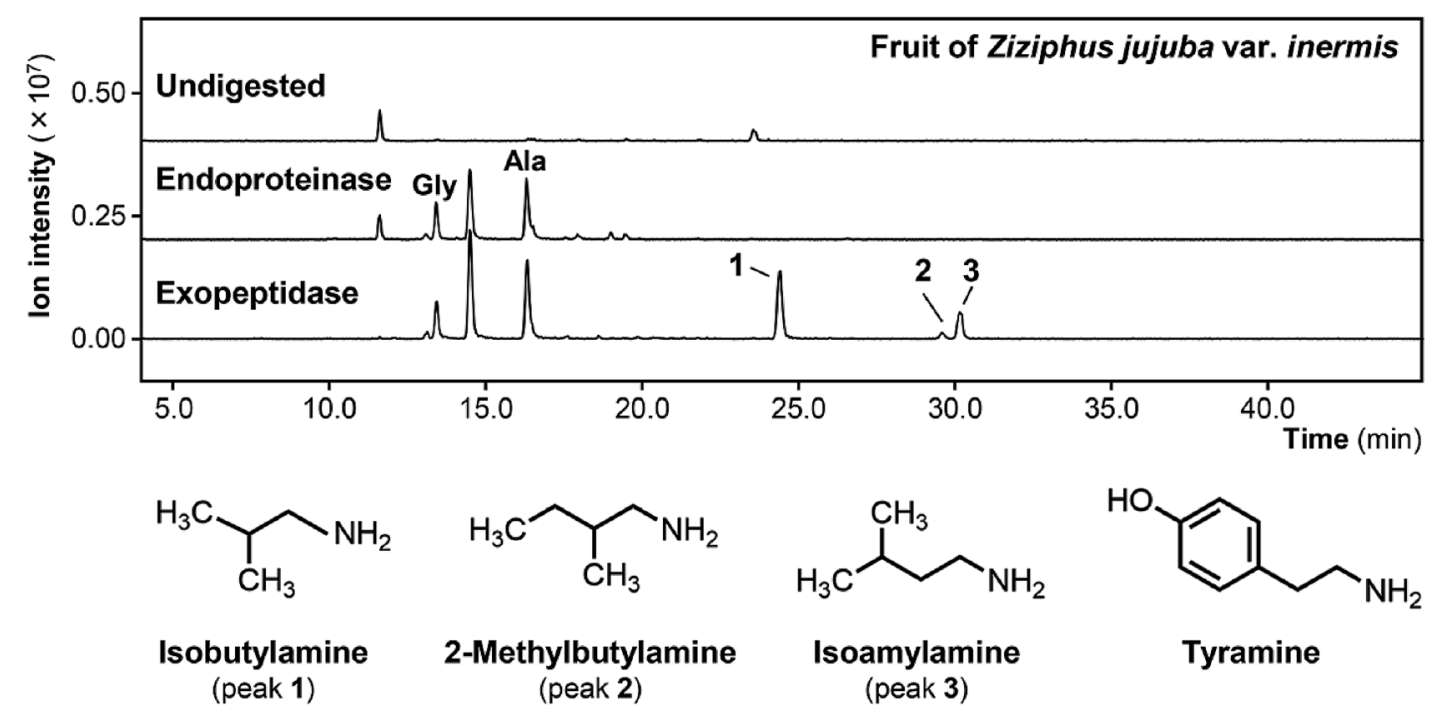

Fig. 2. Total Ion Chromatograms of the in Vitro-Digested Hydrophilic Fraction from the Fruit of Ziziphus jujuba var. inermis (Taiso)

The hydrophilic fraction of the fruit of Ziziphus jujuba var. inermis was digested in vitro and derivatized by AQC, as described in Materials and Methods. Precursor ion scanning was performed in the range of $\mathrm{m} / \mathrm{z}$ from 240 to 275 . Each total ion chromatogram shows the elution of the undigested hydrophilic fraction, an endoproteinase digest, and an exopeptidase digest. $x$ axis, elution time (min); $y$ axis, ion intensity. The peaks at elution times of $24.5,29.7$, and 30.2 min were identified by multiple reaction monitoring (MRM) as isobutylamine, 2-methylbutylamine, and isoamylamine, respectively. Chemical structures of the decarboxylated amino acids present in the digest from the fruit of Ziziphus jujuba var. inermis are depicted under the chromatogram. The peaks at elution times of 13.4 and 16.4 min were identified as glycine (Gly) and alanine (Ala), respectively. The peak between them is not derived from an amino acid and possibly derived from the reagents in the in vitro digestion. 
degraded into amino acids by peptidase digestion.

The protease digests of hydrophilic fractions of crude drug extracts were analyzed by LC-MS/MS. Pyroglutamyl peptides, such as pyroGlu-Leu, which are often present in hydrolysates of foods and Japanese traditional fermented foods, ${ }^{16,17)}$ were not detected in the digests (data not shown). Therefore, compounds with amino group(s) were further investigated.

AQC derivatization of the amines ${ }^{18)}$ was performed to discriminate amines from the other compounds in the digests. When a hydrophilic fraction from the fruit of Ziziphus jujuba var. inermis (Taiso) (Fig. 2, Undigested) was subjected to digestion by endoproteinases (pepsin and pancreatin), several peaks of AQC derivatives appeared in the total ion chromatogram (Fig. 2, Endoproteinase digest) of the precursor ion scan in the range of $\mathrm{m} / \mathrm{z}$ from 240 to 275 . When this digest was further treated with exopeptidases (CPA and LAP), prominent peaks of the AQC derivatives were observed (Fig. 2, Exopeptidase digest) in the same scan range. Precursor ion scanning indicated that several peaks were AQC derivatives of amino acids, for example, glycine and alanine (Fig. 2, peaks Gly and Ala). Furthermore, there were three peaks that were not derivatives of normal amino acids at elution times of 24.5, 29.7, and $30.2 \mathrm{~min}$ in the chromatogram (Fig. 2, peaks 1 to 3 ). In other scan ranges, most peaks observed in the endoproteinase digest of the fruit of Ziziphus jujuba var. inermis disappeared in the exopeptidase digest (data not shown).

On the other hand, peaks of precursor ions were observed in endoproteinase and exopeptidase digests of the fruit of Ziziphus jujuba var. inermis in the scan range of $\mathrm{m} / \mathrm{z}$ from 240 to 500. These peaks correspond to AQC-derivatized amino acids, which were identified by comparison with AQC derivatives of standard amino acids, as described in Materials and Methods. The peak corresponding to alanine was detected, whereas the peaks corresponding to valine, isoleucine, and leucine were not detected in the exopeptidase digest (data not shown). There were a few small peaks of AQC-derivatized compounds that were not identified in the exopeptidase digest in the range of $\mathrm{m} / \mathrm{z}$ from 300 to 500 .

Identification of Decarboxylated Amino Acids in Protease Digests To identify the AQC fragment ions corresponding to the three peaks, product ion scanning was performed. As shown in Fig. 3, the MS spectrum of the peak at $24.5 \mathrm{~min}$ $(\mathrm{m} / \mathrm{z}$ 244.2) showed a shift of 73.0 from the AQC fragment $(m / z$ 171.0) when the collision energy was decreased. These results suggested that the 24.5 -min peak is derived from isobutylamine (molecular weight, 73.14), which is a primary amine as a decarboxylated form of valine.

The product ion scanning of peaks at 29.7 and $30.2 \mathrm{~min}$ (Fig. 3) suggested that these peaks were derived from 2-methylbutylamine or isoamylamine, which could not be discriminated by their MS spectra because the patterns of the fragment ions were the same (Supplementary Figs. 1 and 2). When MRM using standard compounds was applied, it was confirmed that the 29.7- and 30.2-min peaks were attributed to 2-methylbutylamine and isoamylamine, respectively (data not shown). The chemical structures of 2-methylbutylamine and isoamylamine (Fig. 2) show that they are decarboxylated forms of isoleucine and leucine, respectively. In the protease digest from the fruit of Ziziphus jujuba var. inermis, three decarboxylated amino acids were identified, which may be derived from branched-chain amino acids.
Then, in vitro protease digests from the other crude drugs were subjected to precursor ion scanning and examined to determine whether isobutylamine, 2-methylbutylamine, and isoamylamine were present in the digests (Fig. 4). The amounts

A

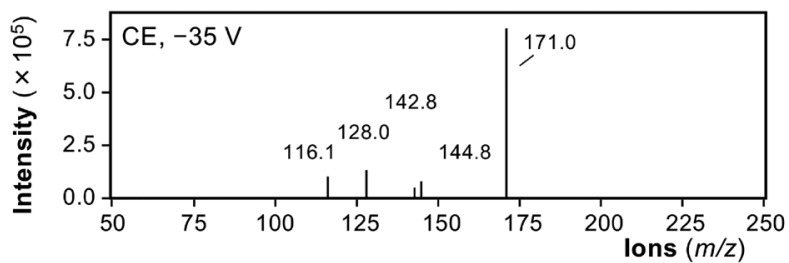

B

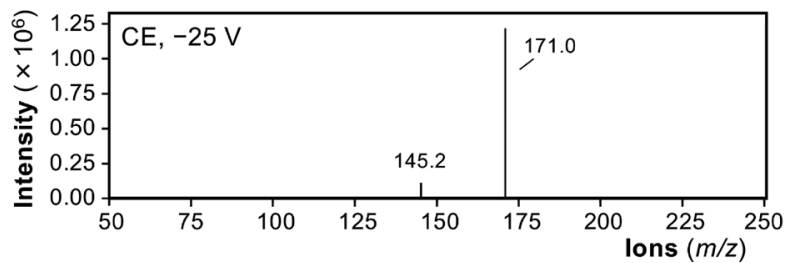

C

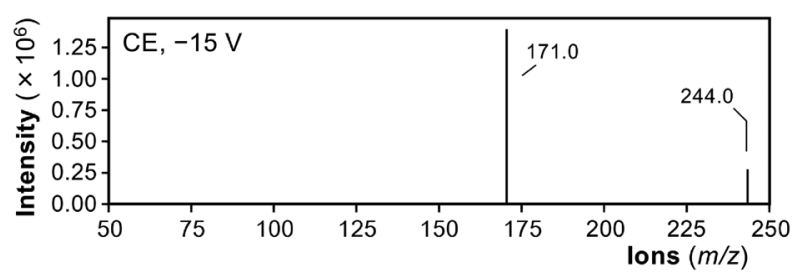

Fig. 3. Identification of Isobutylamine

The AQC fragment ion at the 24.5-min peak of the exopeptidase digest from the fruit of Ziziphus jujuba var. inermis was analyzed. Product ion scanning targeting the AQC-derivative precursor ion $(m / z 244.2)$ was performed, as described in Materials and Methods. (A-C) MS spectra of the product ion scan. The AQC-fragment ion was analyzed by product ion scanning at collision energies of $-35 \mathrm{~V}(\mathrm{~A}),-25 \mathrm{~V}$ (B), and $-15 \mathrm{~V}(\mathrm{C})$. The AQC fragment ion $(\mathrm{m} / \mathrm{z} 171.0)$ was observed in all the ion scanning profiles of the three products. $x$ axis, mass to charge $(\mathrm{m} / \mathrm{z}) ; y$ axis, ion intensity.

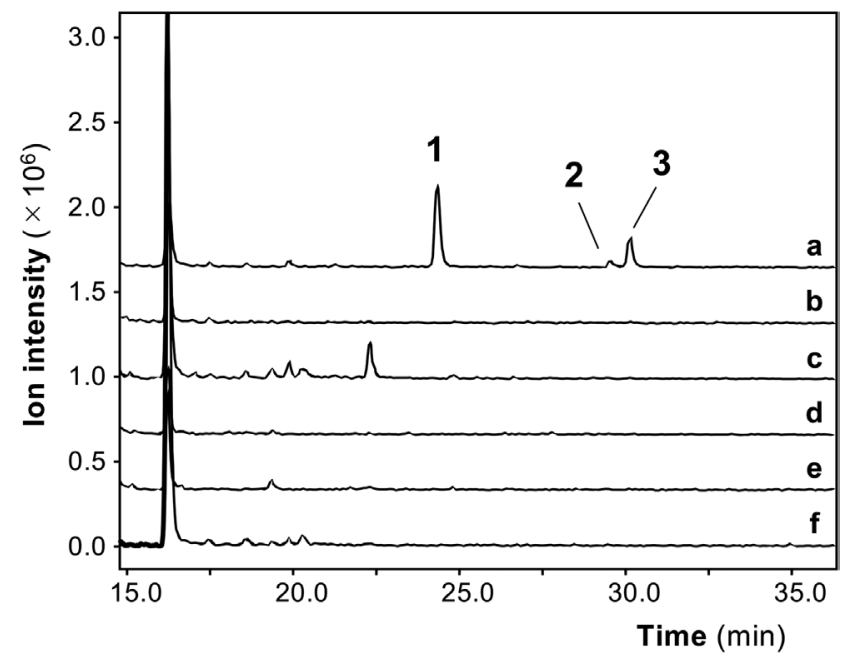

Fig. 4. Precursor Ion Scanning of the in Vitro Protease Digests from Six Crude Drugs

A series of in vitro protease digests from the six crude drugs were derivatized by AQC, as described in Materials and Methods. The AQC derivatives in the exopeptidase digests were analyzed in precursor scan mode in the range of $\mathrm{m} / \mathrm{z}$ from 240 to 275 . Total ion chromatograms are shown. The crude drugs used for this analysis were the fruit of Ziziphus jujuba var. inermis (a), the seed of Ziziphus jujuba var. spinosa (b), the rhizome of Atractylodes macrocephala (c), the rhizome of Atractylodes chinensis rhizome (d), the flower of Artemisia capillaris (e), and the root of Angelica acutiloba (f). $x$ axis, elution time (min); $y$ axis, ion intensity. The numbers 1 to 3 indicate the peaks at elution times of $24.5,29.7$, and $30.2 \mathrm{~min}$, which correspond to isobutylamine, 2-methylbutylamine, and isoamylamine, respectively. 
Table 1. Decarboxylated Amino Acids Derived from Crude Drugs

\begin{tabular}{|c|c|c|c|c|c|}
\hline Crude drug & $\begin{array}{l}\text { (Japanese } \\
\text { name) }\end{array}$ & $\begin{array}{c}\text { Isobutylamine } \\
{[\mathrm{pmol} / \mathrm{mg} \text { fraction }]^{a)}}\end{array}$ & 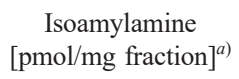 & $\begin{array}{l}\text { 2-Methylbutylamine } \\
{\text { [pmol/mg fraction }]^{a)}}\end{array}$ & $\begin{array}{c}\text { Tyramine } \\
{[\mathrm{pmol} / \mathrm{mg} \text { fraction }]^{a)}}\end{array}$ \\
\hline Fruit of Ziziphus jujuba Miller var. inermis Rehder & Taiso & $69.9 \pm 4.30$ & $18.5 \pm 0.695$ & $3.96 \pm 0.204$ & $0.437 \pm 0.112$ \\
\hline $\begin{array}{l}\text { Seed of Ziziphus jujuba Miller var. spinosa } \mathrm{Hu} \\
\text { ex H. F. Chou }\end{array}$ & Sansonin & - & - & - & $0.327 \pm 0.019$ \\
\hline Rhizome of Atractylodes macrocephala Koidzumi & Byakujutsu & $0.197 \pm 0.019$ & - & - & $1.200 \pm 0.050$ \\
\hline Rhizome of Atractylodes chinensis Koidzumi & Sojutsu & - & - & - & $0.216 \pm 0.004$ \\
\hline Flower of Artemisia capillaris Thunberg & Inchinko & - & - & - & $0.880 \pm 0.040$ \\
\hline Root of Angelica acutiloba Kitagawa & Toki & - & - & - & $0.116 \pm 0.021$ \\
\hline
\end{tabular}

a) The hydrophilic fraction of the extracts of crude drugs was digested by protease, AQC-derivatized, and analyzed using LC-MS/MS, as described in Materials and Methods. The amount of decarboxylated amino acids derived from crude drugs was identified and measured. The content was calculated as each decarboxylated amino acid (pmol) divided by the dried hydrophilic fraction $(\mathrm{mg})$.
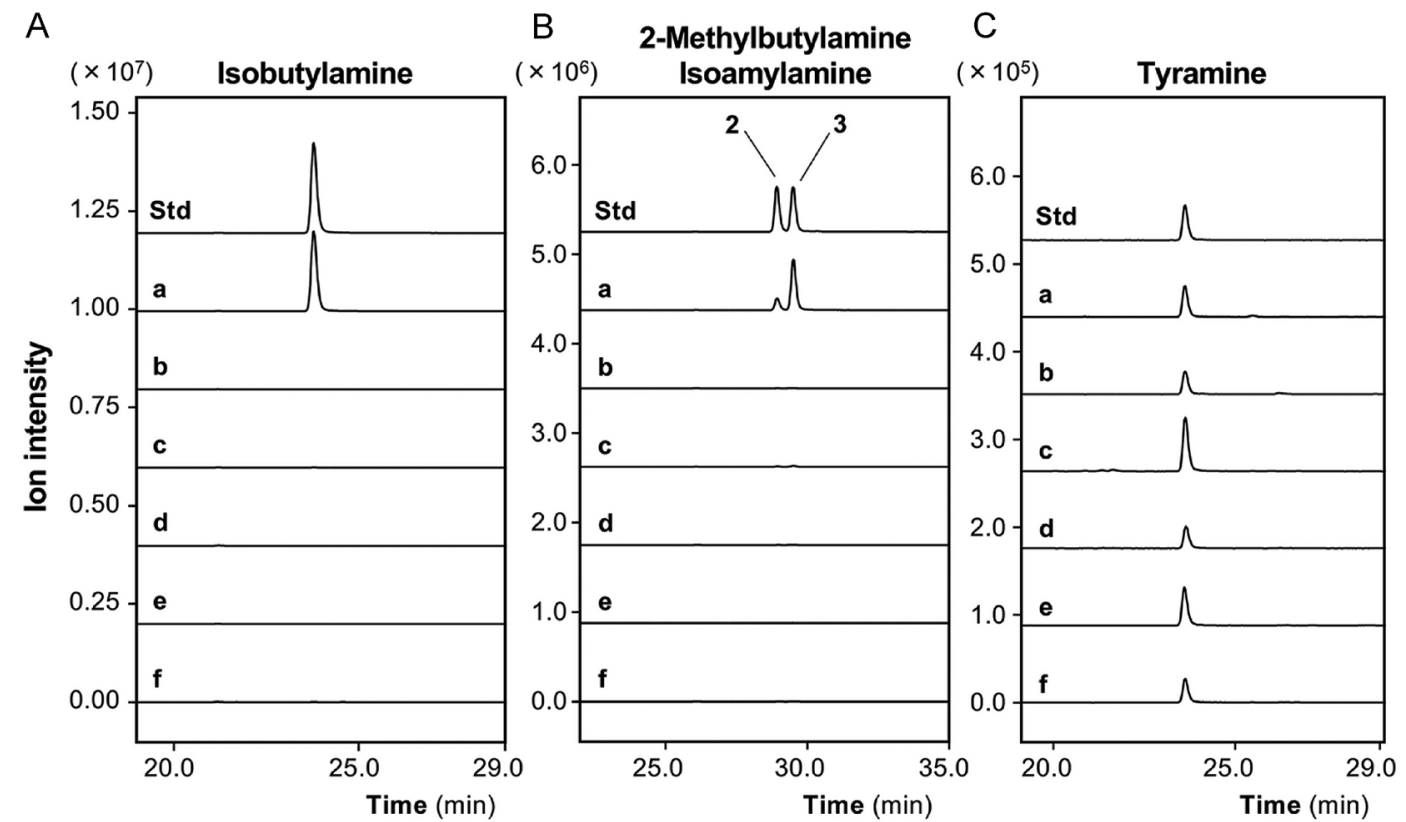

Fig. 5. Detection of Decarboxylated Amino Acids Derived from Six Crude Drugs

The AQC-derivatized decarboxylated amino acids in exopeptidase digests from six crude drugs were detected by LC-MS/MS by MRM, as described in the Materials and Methods. (A) Total ion chromatogram of isobutylamine. (B) Total ion chromatogram of 2-methylbutylamine. isoamylamine. (C) Total ion chromatogram of tyramine. The crude drugs used are the fruit of Ziziphus jujuba var. inermis (a), the seed of Ziziphus jujuba var. spinosa (b), the rhizome of Atractylodes macrocephala (c), the rhizome of Atractylodes chinensis rhizome (d), the flower of Artemisia capillaris (e), and the root of Angelica acutiloba (f). $x$ axis, elution time (min); $y$ axis, ion intensity. Each standard compound was used for the measurement of the content in the exopeptidase digest.

of decarboxylated amino acids were precisely measured in the digests from the crude drugs by MRM (Table 1). Isobutylamine was detected at a high content in the digest from the fruit of Ziziphus jujuba var. inermis, whereas a much lower content was detected in the rhizome of Atractylodes macrocephala. Isoamylamine and 2-methylbutylamine were not detected in the digests from the other crude drugs.

Detection of Tyramine in the Protease Digests from All the Crude Drug Extracts The AQC derivatives of the hydrophilic fraction of other crude drug extracts were further examined. Very small peaks at $23.5 \mathrm{~min}$ in the total ion chromatogram were found in the in vitro protease digests of all the crude drugs used in this study. This AQC fragment ion was expected to be tyramine, which is a decarboxylated form of tyrosine. MRM using tyramine as a standard compound confirmed that the 23.5-min peak was derived from tyramine. The content of tyramine was measured in the digests from the crude drugs by MRM analysis (Fig. 5), which suggested that the content of tyramine was much lower than those of isobu- tylamine, isoamylamine, and 2-methylbutylamine (Table 1).

Suppression of NO Production by Four Decarboxylated Amino Acids in Hepatocytes We examined whether decarboxylated amino acids affect NO production in IL- $\beta$-treated hepatocytes. ${ }^{5)}$ When isobutylamine, which was the most abundant (Table 1), was added to the medium, it decreased IL- $1 \beta$ induced NO production in a dose-dependent manner (Fig. 6). In contrast, the hydrophilic fraction of the extract from the fruit of Ziziphus jujuba var. inermis showed a slight decrease in NO levels. The LDH activity in the medium including each decarboxylated amino acid and the hydrophilic fraction showed much lower LDH activity than that of the whole cell extract (data not shown), suggesting that they were not cytotoxic at the concentrations applied. Similarly, the other three decarboxylated amino acids significantly inhibited NO production without showing cytotoxicity (data not shown). The $\mathrm{IC}_{50}$ values calculated from these data are summarized in Table 2.

In addition, direct NO quenching activity of either decarboxylated amino acid was not observed (data not shown). 


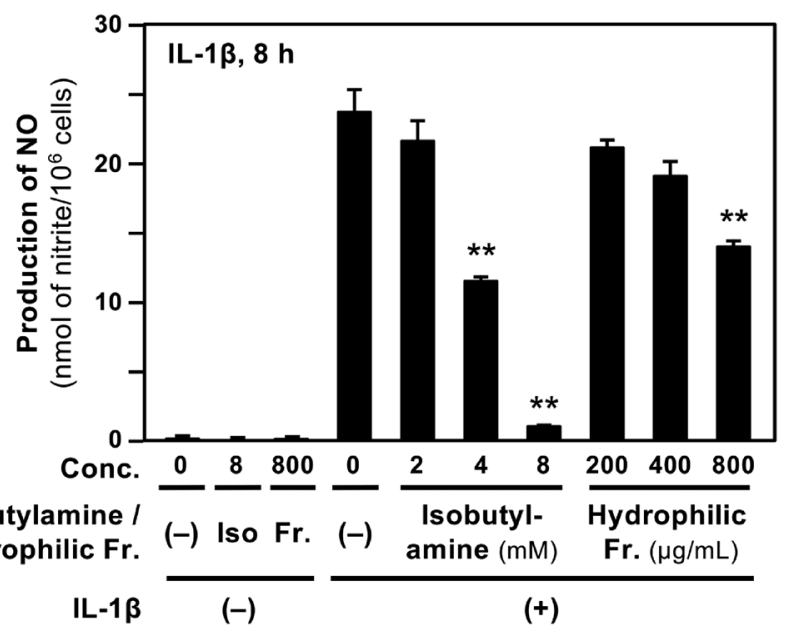

Fig. 6. Isobutylamine Suppresses Nitric Oxide (NO) Production in Rat Hepatocytes

Primary cultured rat hepatocytes were treated with $1 \mathrm{nM}$ IL-1 $\beta$ in the presence or absence of isobutylamine or hydrophilic fraction of the extract from the fruit of Ziziphus jujuba var. inermis for $8 \mathrm{~h}$. The levels of nitrite (a major metabolite of NO) in the medium were measured in triplicate. The values are the mean \pm standard deviation (S.D.) $(n=3) . * * p<0.01$ versus IL-1 $\beta$ alone.

Table 2. Decarboxylated Amino Acids Derived from Crude Drugs and Their Effects on Nitric Oxide Production

\begin{tabular}{lc}
\hline \hline Decarboxylated amino acids & $\mathrm{IC}_{50}[\mathrm{mM}]^{a)}$ \\
\hline Isobutylamine & $4.23 \pm 0.308$ \\
Isoamylamine & $2.42 \pm 0.245$ \\
2-Methylbutylamine & $2.98 \pm 0.339$ \\
Tyramine & $0.833 \pm 0.142$
\end{tabular}

a) The half-maximal inhibitory concentration of nitric oxide production in IL- $1 \beta$ treated hepatocytes (mean \pm standard deviation). At least three experiments using a standard compound were performed to determine these values.

Therefore, the $\mathrm{IC}_{50}$ values of decarboxylated amino acids on NO production were calculated under the criteria described in the Materials and Methods. As summarized in Table 2, the four decarboxylated amino acids exhibited significant decreases in IL- $1 \beta$-induced NO production in hepatocytes. Because the hydrophilic fraction of the extract from the fruit of Ziziphus jujuba var. inermis, which was not digested by protease, showed little suppressing effect on NO production (Fig. 6), its $\mathrm{IC}_{50}$ value could thus not be calculated. Similarly, undigested hydrophilic fraction of the extracts from the other five crude drugs did not significantly suppress NO production (data not shown).

Decarboxylation of Branched-Chain Amino Acids To detect free amino acids in the undigested hydrophilic fraction of the fruit of Ziziphus jujuba var. inermis, this fraction was directly derivatized by AQC and analyzed using LC-MS/MS. This analysis indicated that valine, isoleucine, and leucine were not detected in this fraction (data not shown).

Next, to examine whether branched-chain amino acids are substrates of proteases, valine, isoleucine, and leucine were subjected to in vitro digestion by proteases (endoproteinase and exopeptidase). When calculated from observed peaks of AQC derivatives in the exopeptidase digest, molar ratios of a decarboxylated amino acid to corresponding amino acid were $0.025 \%$ (isobutylamine/valine), $0.022 \%$ (2-methylbutylamine/ isoleucine), and $0.016 \%$ (isoamylamine/leucine). These results imply that traces of branched-chain amino acids were decarboxylated during in vitro protease digestion.

\section{DISCUSSION}

When a crude drug is orally administered, hydrophilic (water-soluble) constituents are degraded by endoproteinases and exopeptidases. LC-MS/MS analyses of in vitro protease digests of hydrophilic constituents from six crude drugs indicated that primary amines were successfully identified after AQC derivatization. The compounds identified were decarboxylated amino acids (i.e., isobutylamine, 2-methylbutylamine, and isoamylamine), which were characteristically detected at a high content in the digest from the fruit of Ziziphus jujuba var. inermis (Table 1). Isobutylamine and 2-methylbutylamine are known as food additives for flavoring, and there is no safety concern with these compounds. ${ }^{25)}$ Furthermore, tyramine, a decarboxylated tyrosine, was identified in the digests from all the crude drugs. Therefore, four decarboxylated amino acids in total were identified as the products after in vitro protease digestion of the crude drugs of Kampo medicine.

It is expected that some compounds in protease digests are pharmacologically active, for example, showing antiinflammatory effects. Many crude drugs and their constituents suppress NO production, which correlates with their anti-inflammatory effects in hepatocytes. ${ }^{7)}$ When the four decarboxylated amino acids were examined, all of them significantly inhibited NO production (Table 2). In contrast, the undigested hydrophilic fraction of the extract from the fruit of Ziziphus jujuba var. inermis showed only a slight decrease in NO production (Fig. 6). The hydrophilic fraction from this crude drug contained protein $(15.9 \mu \mathrm{g}$ per mg fraction in dry weight, $1.59 \%$ ) but mostly consisted of fructose and glucose. It is plausible that the hydrophilic fraction had little effect on NO production because fructose and glucose did not inhibit NO production. ${ }^{1)}$ As shown in Table 2, the $\mathrm{IC}_{50}$ values of the decarboxylated amino acids on NO production ranged from $0.833 \mathrm{mM}$ (tyramine) to $4.23 \mathrm{mM}$ (isobutylamine). The $\mathrm{IC}_{50}$ values of other constituents or drugs were $0.652 \mathrm{mM}$ (chlorogenic acid), ${ }^{1)} 2.89 \mathrm{mM}$ (acetaminophen), ${ }^{24)}$ and $7.01 \mathrm{mM}$ (aspirin $)^{24)}$ in hepatocytes. Comparison of these values implies that decarboxylated amino acids may have comparable potency to those compounds.

In our previous study using hepatocytes, the hydrophilic fraction of many crude drug extracts did not show high potency on NO production. ${ }^{2,3)}$ Similarly, the hydrophilic fraction of the extract from the fruit of Ziziphus jujuba var. inermis had low potency (Fig. 6). In addition, valine, isoleucine, leucine, or tyrosine did not significantly inhibit NO production. ${ }^{11)}$ This study clearly demonstrated that the four decarboxylated amino acids significantly inhibited NO production (Fig. 6). Taken together, both normal amino acids and decarboxylated amino acids, which were produced by the digestion of crude drugs, may be responsible for their anti-inflammatory effects in vivo.

How decarboxylated amino acids are formed during in vitro protease digestion of the hydrophilic fraction of the fruit of Ziziphus jujuba var. inermis has not been elucidated. It is unlikely that free amino acids in the digests are decarboxylated. As above-mentioned, the branched-chain amino acids were not detected in the undigested hydrophilic fraction of the fruit of Ziziphus jujuba var. inermis. When valine, isoleucine, 
and leucine were subjected to in vitro protease digestion, they were hardly decarboxylated, suggesting that the proteases used in this study does not include branched-chain amino acid decarboxylase. Furthermore, decarboxylation of branched-chain amino acids seems to be unfavorable with regards to chemical reactions. It is rather possible that $\mathrm{C}$-terminal decarboxylated amino acid residues of peptides were cleaved during digestion.

The four decarboxylated amino acids identified in this study were detected in the cecum and colon of horses, although bacterial amino acid decarboxylase in the intestine may be involved. ${ }^{26)}$ In humans, the microflora is not formed in the intestine of newborns at birth but is gradually formed by breast milk feeding. ${ }^{27)}$ Isoamylamine and tyramine were detected in the feces of newborns, as well as the breast milk of mothers, within $30 \mathrm{~d}$ after childbirth. ${ }^{27)}$ Recently, the mouse Gm853 gene was found to encode leucine decarboxylase, which catalyzes the reaction to form isoamylamine from leucine and is expressed in the kidney. ${ }^{28)}$ Any other mammalian branched-chain amino acid decarboxylase was not found in the literatures that we have searched to date. It is speculated that both endogenous amino acid decarboxylase and bacterial amino acid decarboxylase may produce primary amines in the body. It is also possible that the fruit of Ziziphus jujuba var. inermis has an enzyme that decarboxylates C-terminal branched-chain amino acid residues of peptides.

Our approach using in vitro protease digestion enabled us to find decarboxylated amino acids showing anti-inflammatory activity. Proteins and peptides in the hydrophilic fraction from crude drug extracts are difficult to investigate because they are degraded and metabolized after oral administration. It is highly possible that the hydrophilic fraction is digested to form protease-resistant peptides that possess a variety of pharmacological activities. Recently, Memarpoor-Yazdi et al. reported that bioactive tripeptides in the protease digest protein from the fruit of Ziziphus jujuba var. inermis. ${ }^{29)}$ They used trypsin and papain (plant protease) for in vitro digestion and found that a protease-resistant tripeptide (Ile-Glu-Arg) competitively inhibited rabbit angiotensin converting enzyme. Therefore, the current study may be applied to pharmacological studies on proteins and peptides and facilitate understanding how crude drugs and Kampo medicine manifest their effects in vivo.

Acknowledgments We thank our students, Mr. Yuma Nishimoto, Mr. Toshinari Ishii, Ms. Nana Toyao, Ms. Yuri Mizuta, Mr. Yuya Kamikawa, Mr. Toshitaka Suematsu, and Mr. Mitsuhiro Aoyagi, for fractionation of crude drugs; Dr. Tetsuya Okuyama for his technical suggestions; and Ms. Noriko Kanazawa for her secretarial assistance. S.S. was supported by the Scholarship Fund for Young/Women Researchers of the Promotion and Mutual Aid Corporation for Private Schools of Japan. This work was supported in part by the Asia-Japan Research Institute of Ritsumeikan Asia-Japan Research Organization, Ritsumeikan University.

Conflict of Interest S.M. performed this study as an undergraduate student of the College of Life Sciences, Ritsumeikan University. The other authors declare no conflict of interest.

Supplementary Materials This article contains supple- mentary materials.

\section{REFERENCES}

1) Ohno N, Yoshigai E, Okuyama T, Yamamoto Y, Okumura T, Sato K, Ikeya Y, Nishizawa M. Chlorogenic acid from the Japanese herbal medicine Kinginka (Flos Lonicerae japonicae) suppresses the expression of inducible nitric oxide synthase in rat hepatocytes. HOAJ Biol., 1, 2 (2012).

2) Fujii A, Okuyama T, Wakame K, Okumura T, Ikeya Y, Nishizawa M. Identification of anti-inflammatory constituents in Phellodendri Cortex and Coptidis Rhizoma by monitoring the suppression of nitric oxide production. J. Nat. Med., 71, 745-756 (2017).

3) Yamauchi Y, Okuyama T, Ishii T, Okumura T, Ikeya $Y$, Nishizawa M. Sakuranetin downregulates inducible nitric oxide synthase expression by affecting interleukin-1 receptor and CCAAT/enhancerbinding protein $\beta$. J. Nat. Med., 73, 353-368 (2019).

4) Ningsih FN, Okuyama T, To S, Nishidono Y, Okumura T, Tanaka K, Ikeya Y, Nishizawa M. Comparative analysis of anti-inflammatory activity of the constituents of the rhizome of Cnidium officinale using rat hepatocytes. Biol. Pharm. Bull., 43, 1867-1875 (2020).

5) Kitade H, Sakitani K, Inoue K, Masu Y, Kawada N, Hiramatsu Y, Kamiyama Y, Okumura $\mathrm{T}$, Ito $\mathrm{S}$. Interleukin $1 \beta$ markedly stimulates nitric oxide formation in the absence of other cytokines or lipopolysaccharide in primary cultured rat hepatocytes but not in Kupffer cells. Hepatology, 23, 797-802 (1996).

6) Colasanti M, Suzuki H. The dual personality of NO. Trends Pharmacol. Sci., 21, 249-252 (2000).

7) Nishizawa M, Okumura T, Ikeya Y. Assessment of antiinflammatory effects of Japanese Kampo medicine and functional foods. Functional Foods in Health and Disease, 9, 79-91 (2019).

8) Hattori M. Intestinal bacteria play a significant role in the medicinal effects of Kampo medicines. Journal of Intestinal Microbiology, 26, 159-169 (2012). doi: 10.11209/jim.26.159

9) Matsumoto M, Ishige A, Yazawa Y, Kondo M, Muramatsu K, Watanabe K. Promotion of intestinal peristalsis by Bifidobacterium spp. capable of hydrolysing sennosides in mice. PLOS ONE, 7, e31700 (2012).

10) Tanemoto R, Okuyama T, Matsuo H, Okumura T, Ikeya Y, Nishizawa M. The constituents of licorice (Glycyrrhiza uralensis) differentially suppress nitric oxide production in interleukin-1 $\beta$-treated hepatocytes. Biochem. Biophys. Rep., 2, 153-159 (2015).

11) Miki H, Tokuhara K, Oishi M, Tanaka Y, Nakatake R, Ueyama Y, Kaibori M, Nishizawa M, Okumura T, Kon M. Elental ${ }^{\mathbb{R}}$ amino acid component has protective effects on primary cultured hepatocytes and a rat model of acute liver injury. Nutr. Res., 42, 71-84 (2017).

12) Elbarbary HA, Ejima A, Sato K. Generation of antibacterial peptides from crude cheese whey using pepsin and rennet enzymes at various pH conditions. J. Sci. Food Agric., 99, 555-563 (2019).

13) Chen L, Ejima A, Gu R, Lu J, Cai M, Sato K. Presence of exopeptidase-resistant and susceptible peptides in a bacterial protease digest of corn gluten. J. Agric. Food Chem., 67, 11948-11954 (2019).

14) Sato K. Structure, content, and bioactivity of food-derived peptides in the body. J. Agric. Food Chem., 66, 3082-3085 (2018).

15) Nwachukwu ID, Aluko RE. Structural and functional properties of food protein-derived antioxidant peptides. J. Food Biochem., 43, e12761 (2019)

16) Oishi M, Kiyono T, Sato K, Tokuhara K, Tanaka Y, Miki H, Nakatake R, Kaibori M, Nishizawa M, Okumura T, Kon M. pyroGluLeu inhibits the induction of inducible nitric oxide synthase in interleukin- $1 \beta$-stimulated primary cultured rat hepatocytes. Nitric Oxide, 44, 81-87 (2015).

17) Shirako S, Kojima Y, Tomari N, Nakamura Y, Matsumura Y, Ikeda $\mathrm{K}$, Inagaki N, Sato K. Pyroglutamyl leucine, a peptide in fermented foods, attenuates dysbiosis by increasing host antimicrobial peptide. NPJ Sci. Food, 3, 18 (2019). 
18) Ejima A, Nakamura M, Suzuki YA, Sato K. Identification of foodderived peptides in human blood after ingestion of corn and wheat gluten hydrolysates. J. Food Bioact., 2, 104-111 (2018).

19) The Committee on the Japanese Pharmacopoeia. The Japanese Pharmacopoeia, 18th edn., The Minister of Health, Labour and Welfare, Tokyo (2021).

20) Ishii $T$, Okuyama $T$, Noguchi $N$, Nishidono $Y$, Okumura $T$, Kaibori M, Tanaka K, Terabayashi S, Ikeya Y, Nishizawa M. Antiinflammatory constituents of Atractylodes chinensis rhizome improve glomerular lesions in immunoglobulin A nephropathy model mice. J. Nat. Med., 74, 51-64 (2020)

21) Asai $T$, Takahashi $A$, Ito $K$, Uetake $T$, Matsumura $Y$, Ikeda $K$, Inagaki N, Nakata M, Imanishi Y, Sato K. Amount of collagen in the meat contained in Japanese daily dishes and the collagen peptide content in human blood after ingestion of cooked fish meat. J. Agric. Food Chem., 67, 2831-2838 (2019).

22) Kanemaki T, Kitade H, Hiramatsu Y, Kamiyama Y, Okumura T. Stimulation of glycogen degradation by prostaglandin $E_{2}$ in primary cultured rat hepatocytes. Prostaglandins, 45, 459-474 (1993).

23) Green LC, Wagner DA, Glogowski J, Skipper PL, Wishnok JS, Tannenbaum SR. Analysis of nitrate, nitrite, and $\left.{ }^{[5} \mathrm{N}\right]$ nitrate in biological fluids. Anal. Biochem., 126, 131-138 (1982).

24) Inaba $\mathrm{H}$, Yoshigai $\mathrm{E}$, Okuyama $\mathrm{T}$, Murakoshi M, Sugiyama K,
Nishino H, Nishizawa M. Antipyretic analgesic drugs have different mechanisms for regulation of the expression of inducible nitric oxide synthase in hepatocytes and macrophages. Nitric Oxide, 44, 61-70 (2015).

25) Food Safety Commission of Japan. Isobutylamine, isopropylamine, sec-butylamine, propylamine, hexylamine, pentylamine and 2-methylbutylamine (flavoring substances). Food Saf. (Tokyo), 7, 54-55 (2019)

26) Bailey SR, Marr CM, Elliott J. Identification and quantification of amines in the equine caecum. Res. Vet. Sci., 74, 113-118 (2003).

27) Suárez L, Moreno-Luque M, Martínez-Ardines I, González N, Campo P, Huerta-Cima P, Sánchez M. Amine variations in faecal content in the first weeks of life of newborns in relation to breastfeeding or infant formulas. Br. J. Nutr., 122, 1130-1141 (2019).

28) Lambertos A, Ramos-Molina B, Cerezo D, López-Contreras AJ, Peñafiel R. The mouse $G m 853$ gene encodes a novel enzyme: leucine decarboxylase. Biochim. Biophys. Acta Gen. Subj., 1862, 365-376 (2018).

29) Memarpoor-Yazdi M, Zare-Zardini H, Mogharrab N, Navapour L. Purification, characterization and mechanistic evaluation of angiotensin converting enzyme inhibitory peptides derived from Zizyphus jujuba fruit. Sci. Rep., 10, 3976 (2020). 